\title{
Peculiarities of acousto-optic diffraction at circularly polarized acoustic waves. Determination of elasto-optic coefficients coupled with shear waves
}

\author{
Kostyrko M., Orykhivskyi I., Skab I. and Vlokh R.
}

O. G. Vlokh Institute of Physical Optics, 23 Dragomanov Street, 79005 Lviv, Ukraine, vlokh@ifo.lviv.ua

Received: 07.10 .2020

\begin{abstract}
We develop a new approach for determining some of elasto-optic coefficients ( $p_{i j}$ with $i=1 \ldots 6$ and $\left.j=4,5\right)$ basing on Dixon-Cohen method and acousto-optic diffraction at circularly polarized acoustic waves. Particular cases of crystals that belong to trigonal system and some symmetry groups of tetragonal and hexagonal systems are analyzed. We find that the effective elasto-optic coefficients are different for the alternative cases of diffractions at either right- or left-handed circularly polarized acoustic waves that propagate along $Z$ axis in crystals. One can determine in this way the coefficients $p_{44}$ and $p_{45}$ at the anisotropic diffraction in the crystals belonging to point symmetry groups $4,4 / \mathrm{m}, \overline{4}, 6,6 / \mathrm{m}$ and $\overline{6}$. For the crystals belonging to symmetry groups $32,3 \mathrm{~m}$ and $\overline{3} \mathrm{~m}$, it is possible to determine the coefficients $p_{14}$ and $p_{44}$ respectively at the isotropic and anisotropic diffractions. Finally, for the crystals described by the groups 3 and $\overline{3}$, one can determine separately the four coefficients $p_{44}, p_{45}, p_{25}$ and $p_{14}$ following from the anisotropicdiffraction data and the two coefficients $p_{14}$ and $p_{25}$ following from the isotropicdiffraction data.
\end{abstract}

Keywords: circularly polarized acoustic waves, acousto-optic diffraction, effective elasto-optic coefficients

UDC: $535.421+535.551+534.27$

\section{Introduction}

Acousto-optic (AO) diffraction is a well known phenomenon, which consists in interaction of optical wave with the phase grating of refractive index caused by acoustic wave (AW) via elastooptic effect [1]. The efficiency of AO Bragg diffraction is given by the relation

$$
\eta=\sin ^{2}\left(\frac{\pi}{\lambda_{0} \cos \Theta_{B}} \sqrt{\frac{P_{a c} L}{2 H} M_{2}}\right),
$$

or, under the condition $\eta \ll 1$, by its simplified version,

$$
\eta=\frac{\pi^{2} L}{2 \lambda_{0}^{2} H \cos \Theta_{B}} M_{2} P_{a c} .
$$

Here $\lambda_{0}$ is the wavelength of optical radiation in vacuum, $\Theta_{B}$ the Bragg angle, $P_{a c}$ the power of the AW, $L$ the length of $\mathrm{AO}$ interaction, $H$ the width of piezoelectric transducer, and $M_{2}$ the $\mathrm{AO}$ figure of merit. In fact, the diffraction efficiency is proportional to the AW power, where the proportionality coefficient is just the AO figure of merit. The latter is defined by a set of constitutive coefficients: 


$$
M_{2}=\frac{n_{i}^{3} n_{d}^{3} p_{e f f}^{2}}{\rho v^{3}},
$$

where $n_{i}$ and $n_{d}$ denote the refractive indices of respectively incident and diffracted optical waves, $p_{\text {eff }}$ implies the effective elasto-optic coefficient (EEC), $v$ the AW velocity, and $\rho$ the material density. The other parameters entering Eqs. (1) and (2) are geometric or they depend on the acoustic and optical wavelengths, together with phase-matching conditions [2].

Hence, the efficiency of AO diffraction depends mainly on the material properties. Basing on Eqs. (2) and (3), Dixon R. W. and Cohen M. G. have developed an AO method for determining elasto-optic coefficients [3]. In general, it involves the studies of efficiency of AO diffraction in a given material with respect to the efficiency referred to some standard material. For example, one can determine the elasto-optic coefficients $p_{i j}$ (with $i, j=1,2,3$ ) using the diffraction at longitudinal AWs, while the coefficients $p_{i i}(i=4,5,6)$ can be measured basing on the diffraction at transverse AWs. The other coefficients are usually combined with each other so that they can be evaluated when utilizing a number of interaction geometries and different crystal cuts. As an example, the coefficients $p_{14}, p_{25}, p_{44}$ and $p_{45}$ are usually superimposed with the other coefficients even for such high-symmetry optically uniaxial crystals as those belonging to the point symmetry groups 3 and $\overline{3}$.

Notice that excitation of circular AWs produces shear mechanical deformations. For instance, the deformation components $e_{4}$ and $e_{5}$ arise when the AW propagates along $Z$ axis. These components can lead to AO coupling that depend upon the elasto-optic coefficients $p_{i j}(i=1 \ldots 6$ and $j=4,5$ ). Recently, we have reported that the case of diffraction of circularly polarized optical waves at AWs in optically active crystals represents a separate area of acousto-optics [4-6]. In particular, when the circular optical waves represent eigenwaves in a material medium, one can consider the isotropic and anisotropic types of AO coupling between two left-handed (LH) (or right-handed (RH)) optical waves or, alternatively, between the RH and LH optical waves. This situation can happen, e.g., in the crystals that belong to gyrotropic cubic point symmetry groups or at the optical wavelength that corresponds to a so-called isotropic point in anisotropic crystals [7]. If the isotropic approximation [6] holds true, the same situation can also be realized in the vicinity of optic axes.

From this point of view, the situation described above is very similar to the AO interaction between linearly polarized optical eigenwaves, i.e. the interaction between the two ordinary (or extraordinary) waves or between the ordinary and extraordinary waves, under condition that the AWs are circularly polarized. As far as we know, the diffraction at the circular AWs has not yet been analyzed in the literature. In the present work, we will show that, in crystals of some particular symmetries, the diffractions occurring at the RH and LH AWs can differ from each other. Moreover, we will demonstrate that it is possible to determine some of the elasto-optic coefficients with the Dixon-Cohen method, when using the circular AWs.

\section{Results and discussion}

Let us consider propagation of a circularly polarized AW along the acoustic axis in a crystal. For example, this can be a direction of four-fold symmetry axis in paratellurite. The transverse AWs propagating along this direction (i.e., along the $Z$ axis) have the same velocities that do not depend on the orientation of displacement vector in the $X Y$ plane. Hence, these waves suffer no linear 
acoustic birefringence. The circular acoustic birefringence, which is caused by the acoustic activity, can be ignored since it cannot manifest itself in our case. When the incident optical wave propagates along the $X$ (or $Y$ ) axis and diffracts at a thick acoustic grating in tangential Bragg regime, one can consider the two alternative interaction planes, $X Z$ and $Y Z$ (see Fig. 1).

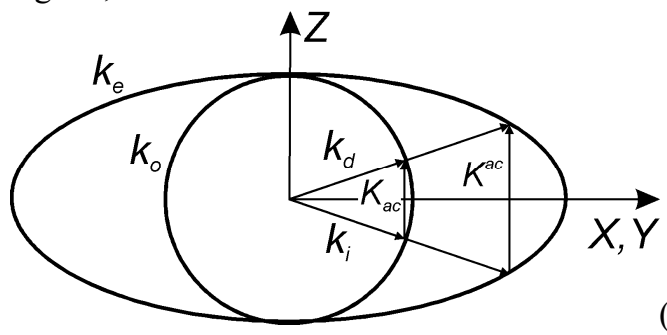

(a)

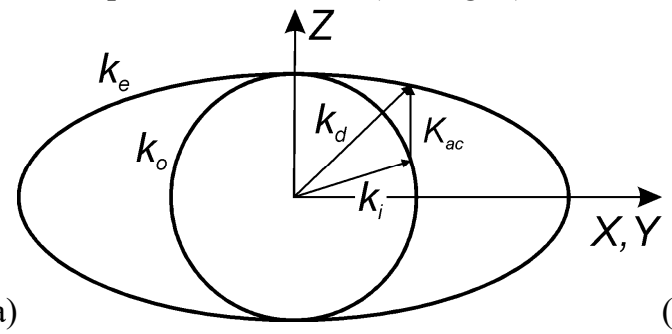

(b)

Fig. 1. Schematic view of $A O$ interactions in optically positive crystals, which arise in the cases of isotropic (a) and anisotropic (b) diffractions.

The components of deformation tensor for the RH and LH AWs can be represented by the systems of equations

$$
\left\{\begin{array} { l } 
{ e _ { 5 } ^ { R H } = - e _ { 0 } \operatorname { c o s } \delta } \\
{ e _ { 4 } ^ { R H } = e _ { 0 } \operatorname { s i n } \delta }
\end{array} \text { and } \left\{\begin{array}{l}
e_{5}^{L H}=e_{0} \cos \delta \\
e_{4}^{L H}=e_{0} \sin \delta
\end{array}\right.\right.
$$

where $e_{0}$ is the unit amplitude of deformation and $\delta$ its phase. In the case of isotropic diffraction inside the $X Z$ and $Y Z$ interaction planes, the EEC is determined by the elasto-optic coefficients $p_{14}$, $p_{15}, p_{24}, p_{25}, p_{34}$ and $p_{35}$, which are equal to zero for the symmetry group 422 . Thus, the isotropic AO interaction (see Fig. 1a) with the circular AW in this experimental geometry cannot be implemented in the paratellurite crystals.

Consider now the anisotropic diffraction (see Fig. 1b) of the ordinary incident optical wave at the RH AW inside the $X Z$ interaction plane. Then the system of equations for the electric-field components of the diffracted wave can be written as

$$
\left\{\begin{array}{l}
E_{1}=\Delta B_{12} D_{2}=\left(p_{64} e_{4}^{R H}+p_{65} e_{5}^{R H}\right) D_{2}=0 \\
E_{3}=\Delta B_{32} D_{2}=\left(p_{44} e_{4}^{R H}+p_{45} e_{5}^{R H}\right) D_{2}=p_{44} \sin \delta D_{2}
\end{array},\right.
$$

where $\Delta B_{i j}$ denotes the increment of optical impermeability tensor and $D_{j}$ is the unit electrical induction of the incident optical wave. After averaging the phase over period $(\bar{\delta}=\pi / 4)$, one gets the EEC equal to $p_{\text {eff }}=\frac{\sqrt{2}}{2} p_{44}$, since the relations $p_{45}, p_{64}, p_{65}=0$ hold true. Under the same diffraction conditions, we still have the same EEC for the case of LH AW.

At the anisotropic diffraction of ordinary incident optical wave at the RH AW inside the interaction plane $Y Z$, the electric field of the diffracted optical wave reads as

$$
\left\{\begin{array}{l}
E_{2}=\Delta B_{21} D_{1}=\left(p_{64} e_{4}^{R H}+p_{65} e_{5}^{R H}\right) D_{1}=0 \\
E_{3}=\Delta B_{31} D_{1}=\left(p_{54} e_{4}^{R H}+p_{55} e_{5}^{R H}\right) D_{1}=-p_{55} \cos \delta D_{1}
\end{array} .\right.
$$

Since we have $p_{55}=p_{44}$, the EEC is equal to $p_{\text {eff }}=-\frac{\sqrt{2}}{2} p_{44}$. The EEC obtained for the alternative case of diffraction at the LH AW is equal to $p_{\text {eff }}=\frac{\sqrt{2}}{2} p_{44}$, i.e. the module of the EEC remains the same. Hence, the EEC modules are the same for the $X Z$ and $Y Z$ interaction planes in 
$\mathrm{TeO}_{2}$. In other words, these parameters are invariant under changing sign of the circular $\mathrm{AW}$.

Nonetheless, the AO figures of merit can differ for the cases of diffractions at the $\mathrm{LH}$ and $\mathrm{RH}$ AWs, since these waves differ by their propagation velocity due to acoustic activity. Then it is not necessary to excite the circular AW for determining the $p_{44}$ coefficient or, in some other terms, it is enough to excite one of the transverse waves. The same is true for the symmetry groups $4 / \mathrm{mmm}$, $4 \mathrm{~mm}, \overline{4} 2 \mathrm{~m}, 622,6 \mathrm{~mm}, 6 / \mathrm{mmm}$ and $\overline{6} \mathrm{~m} 2$ that belong to either tetragonal or hexagonal systems.

Let us now consider the crystals belonging to the symmetry groups $4,4 / \mathrm{m}, \overline{4}, 6,6 / \mathrm{m}$ and $\overline{6}$, and the case of anisotropic diffraction in the geometry illustrated in Fig. 1b. The electric field of the diffracted wave is given by

$$
E_{3}=\Delta B_{32} D_{2}=\left(p_{44} e_{4}^{R H}+p_{45} e_{5}^{R H}\right) D_{2}=\frac{\sqrt{2}}{2}\left(p_{44}-p_{45}\right) D_{2}
$$

when the optical wave diffracts at the RH AW inside the $X Z$ plane. The diffraction at the LH AW under the same conditions yields in the relation

$$
E_{3}=\Delta B_{32} D_{2}=\left(p_{44} e_{4}^{R H}+p_{45} e_{5}^{R H}\right) D_{2}=\frac{\sqrt{2}}{2}\left(p_{44}+p_{45}\right) D_{2} .
$$

Then the EEC is equal to $p_{e f f}=\frac{\sqrt{2}}{2}\left(p_{44}-p_{45}\right)$ in the first case, while we have $p_{\text {eff }}=\frac{\sqrt{2}}{2}\left(p_{44}+p_{45}\right)$ in the second case. Hence, the two above measurements can enable determining separately the coefficients $p_{44}$ and $p_{45}$. Moreover, here the diffraction efficiencies differ for the cases of diffractions at the RH and LH AWs, since they are determined by different EECs.

Let us consider the diffraction at the circular AW in the crystals that belong to the trigonal groups $32,3 \mathrm{~m}$ and $\overline{3} \mathrm{~m}$. The electric field of the optical wave diffracted at the RH AW in the case when isotropic interaction between the ordinary optical waves occurs inside the $X Z$ plane is as follows:

$$
E_{2}=\Delta B_{22} D_{2}=\left(p_{24} e_{4}^{R H}+p_{25} e_{5}^{R H}\right) D_{2}=-\frac{\sqrt{2}}{2} p_{14} D_{2}, p_{e f f}=-\frac{\sqrt{2}}{2} p_{14} .
$$

The EEC modules remain the same when the isotropic interaction inside the $Y Z$ plane occurs or when the optical wave diffracts at the LH AW. Then one can determine the elasto-optic coefficient $p_{14}$. Finally, the EEC is equal to zero at the isotropic interaction between the extraordinary optical waves. The reason is the absence of appropriate elasto-optic coefficients in the four-rank tensor.

Let us proceed to the cases of anisotropic diffraction inside the $X Z$ and $Y Z$ planes in the crystals belonging to the symmetry groups mentioned above. Let the optical waves diffract at the RH or LH AWs. Then the EEC is defined as $p_{\text {eff }}=\frac{\sqrt{2}}{2}\left(p_{14}^{2} \sin ^{2} \varphi+p_{44}^{2} \cos ^{2} \varphi\right)^{1 / 2}$, where $\varphi$ is the angle between the $X$ (or $Y$ ) axis and the wave vector of incident optical wave. Having determined the $p_{14}$ coefficient value in the isotropic-diffraction study, one can also found the $p_{44}$ coefficient.

Finally, we consider the crystals that belong to the point symmetry groups 3 and $\overline{3}$. Let the isotropic interaction between the ordinary optical waves take place inside the $X Z$ and $Y Z$ planes. The EEC modules calculated for the cases of diffractions at the RH and LH AWs read respectively as

$$
\left|p_{e f f}\right|=\frac{\sqrt{2}}{2}\left(p_{25}+p_{14}\right) \text { and }\left|p_{e f f}\right|=\frac{\sqrt{2}}{2}\left(p_{25}-p_{14}\right) \text {. }
$$


This fact enables one to determine the elasto-optic coefficients $p_{14}$ and $p_{25}$ separately. Note that the EECs and, hence, the efficiencies of AO diffractions differ for the cases of interactions with the RH and LH AWs. On the other hand, the EEC is equal to zero when the extraordinary optical waves interact with each other and with circular AWs.

The EEC for the case of anisotropic interaction inside the $X Z$ plane and diffraction at the $\mathrm{RH}$ $\mathrm{AW}$ is as follows:

$$
p_{\text {eff }}=\frac{\sqrt{2}}{2}\left(\left(p_{44}-p_{45}\right)^{2} \cos ^{2} \varphi+\left(p_{25}-p_{14}\right)^{2} \sin ^{2} \varphi\right) .
$$

For the $Y Z$ plane we have

$$
p_{\text {eff }}=\frac{\sqrt{2}}{2}\left(\left(p_{44}+p_{45}\right)^{2} \cos ^{2} \varphi+\left(p_{25}-p_{14}\right)^{2} \sin ^{2} \varphi\right) .
$$

Notice that the EECs differ for the $X Z$ and $Y Z$ interaction planes.

The EEC obtained for the cases of diffractions at the LH AW are given by

$$
p_{\text {eff }}=\frac{\sqrt{2}}{2}\left(\left(p_{44}+p_{45}\right)^{2} \cos ^{2} \varphi+\left(p_{25}+p_{14}\right)^{2} \sin ^{2} \varphi\right)
$$

for the $X Z$ plane and

$$
p_{\text {eff }}=\frac{\sqrt{2}}{2}\left(\left(p_{44}-p_{45}\right)^{2} \cos ^{2} \varphi+\left(p_{25}+p_{14}\right)^{2} \sin ^{2} \varphi\right)
$$

for the $Y Z$ plane. As a consequence, one can determine the four coefficients $p_{44}, p_{45}, p_{25}$ and $p_{14}$ separately by determining the combinations of coefficients given by Eqs. (11)-(14). Notice that, again, the EECs differ for the cases of AO interactions with the RH and LH AWs.

To end up our discussion, we would like to remind that the circular AWs can be excited in different ways, e.g., when using acoustic quarter-wave plates [8] or a Fresnel parallelepiped [9]. However, a known effect of conical refraction usually manifests itself in the trigonal crystals whenever the circular AWs propagate along the three-fold axis. The angle of the internal conical refraction depends on relationships among the elastic-stiffness coefficients $C_{i j}$. It can vary notably for different crystals. For instance, this angle can approach zero (e.g., in $\mathrm{Pb}_{5} \mathrm{Ge}_{3} \mathrm{O}_{11}$, since we have $C_{14} \approx 0[10]$ ) or reach high enough values (e.g., $17.17 \mathrm{deg}$ in quartz or even $30.75 \mathrm{deg}$ in calcite [11]). In the case of internal conical acoustic refraction, a conically shaped acoustic energy walkoff arises, so that the orientation of linear polarization of the AW reveals azimuthal dependence on the angle $\pi$ around the ring of refraction. Therefore, special requirements must be met in order to satisfy the conditions necessary for the AO diffraction at the circular AWs in such crystals. Namely, in the case of tangential Bragg AO interactions, the numerical aperture of the incident optical beam should be large enough for covering the aperture of a conical acoustic beam.

\section{Conclusion}

Issuing from the results of the present work, one can conclude that the AO diffraction at the circular AWs can be considered as an additional approach, which is useful when determining the elasto-optic coefficients via the Dixon-Cohen technique. Using this approach, one can find separately some of the elasto-optic coefficients ( $p_{i j}$ with $i=1 \ldots 6$ and $\left.j=4,5\right)$. In particular, one can measure the coefficients $p_{44}$ and $p_{45}$ under conditions of anisotropic diffraction in the crystals belonging to the symmetry groups $4,4 / \mathrm{m}, \overline{4}, 6,6 / \mathrm{m}$ and $\overline{6}$. For the crystals that belong to the trigonal symmetries $32,3 \mathrm{~m}$ and $\overline{3} \mathrm{~m}$, it is possible to find the coefficients $p_{14}$ and $p_{44}$ respectively at the isotropic and anisotropic diffractions. For the crystals described by the groups 3 and $\overline{3}$, one can find separately the four coefficients $p_{44}, p_{45}, p_{25}$ and $p_{14}$, using the studies of anisotropic diffraction. Finally, the coefficients $p_{14}$ and $p_{25}$ for the same crystals can be evaluated following 
from the isotropic-diffraction data. It is also worth noting that the EECs can be defined by different relationships for the cases of diffraction either at the RH AW or at the LH AW.

\section{Acknowledgement.}

The authors acknowledge financial support of the present study by the Ministry of Education and Science of Ukraine (the Projects \#0120U102031 and \#0118U003899).

\section{References}

1. Magdich L N and Molchanov V Ya, Acoustooptic devices and their application. Gordon and Breach Science Publ. (1989).

2. Balakshiy V I, Parygin V N and Chirkov L E, Physical principles of acousto-optics. Moscow: Radio i Svyaz (1985).

3. Dixon R W and Cohen M G, 1966. A new technique for measuring magnitudes of photoelastic tensors and its application to lithium niobate. Appl. Phys. Lett. 8: 205-207.

4. Mys O, Martynyuk-Lototska I, Pogodin A, Dudok T, Adamenko D, Krupych O, Skab I and Vlokh $\mathrm{R}, 2019$. Acousto-optic interaction between circularly polarized optical eigenwaves: example of $\mathrm{AgGaS}_{2}$ crystals. Appl. Opt. 58: 6012-6018.

5. Mys O, Adamenko D, Skab I and Vlokh R, 2019. Anisotropy of acousto-optic figure of merit for the collinear diffraction of circularly polarized optical waves at the wavelength of isotropic point in $\mathrm{AgGaS}_{2}$ crystals. Ukr. J. Phys. Opt. 20: 73-80.

6. Mys O, Martynyuk-Lototska I, Kostyrko M and Vlokh R, 2020. Anisotropy of acousto-optic figure of merit for $\mathrm{Pb}_{5} \mathrm{Ge}_{3} \mathrm{O}_{11}$ crystals in the case of interaction between circularly polarized optical waves. Appl. Opt. (submitted).

7. Hobden M V, 1968. Optical activity in a non-enantiomorphous crystal: $\mathrm{AgGaS}_{2}$. Acta Cryst. A. 24: 676-680.

8. Auld B A, Quate C F, Shaw H J and Winslow D K, 1966. Acoustic quarterwave plates at microwave frequencies. Appl. Phys. Lett. 9: 436-438.

9. Plicque F, Feipier J and Zarembovitc A, 1970. On a method of generating ultrasonic circularly polarized waves. J. Acoust. Soc. Amer. 47: 168-171.

10. Yamada T, Iwasaki H and Niizeki N, 1972. Elastic and piezoelectric properties of ferroelectric 5 PbO-3GeO 2 crystals. J. Appl. Phys. 43: 771-775.

11. Sirotin Yu I and Shaskolskaya M P, Fundamentals of crystal physics. Moscow: Mir Publishers (1982).

Kostyrko M., Orykhivskyi I., Skab I. and Vlokh R. 2020. Peculiarities of acousto-optic diffraction at circularly polarized acoustic waves. Determination of elasto-optic coefficients coupled with shear waves. Ukr.J.Phys.Opt. 21: 201 - 206. doi: 10.3116/16091833/21/4/201/2020

Анотація. Розроблено новий підхід до визначення деяких із еластооптичних коефіцієнтів ( $p_{i j}$ iз $i=1 \ldots 6$ та $\left.j=4,5\right)$ на основі методу Діксона-Коена та акустооптичної дифракиії на циркулярно поляризованих акустичних хвилях. Проаналізовано конкретні випадки кристалів, що належать до тригональної системи і деяких груп симетрії тетрагональноі та гексагональної систем. Виявлено, щчо ефективні еластооптичні коефіџієнти різні для альтернативних випадків дифракиії на правих або лівих акустичних хвилях, які поширюються вздовж осі $Z$ у кристалах. Так можна визначити коефіцієнти $p_{44}$ i $p_{45}$ при анізотропній дифракиії в кристалах, що належсать до точкових груп симетрії 4, 4/m, $\overline{4}, 6$, 6/m i $\overline{6}$. Для кристалів, щзо належать до груп симетрії $32,3 m$ i $\overline{3} m$, можна знайти коефіцієнти $p_{14}$ і $p_{44}$ відповідно при ізотропній і анізотропній дифракиії. Нарешті, для кристалів, що описуються групами $3 i \overline{3}$, можна окремо визначити чотири коефічієнти $p_{44}, p_{45}, p_{25}$ і $p_{14}$ на підставі даних для анізотропної дифракиії і два коефіцієнти $p_{14}$ і $p_{25}$ на підставі даних для ізотропної дифракиії. 